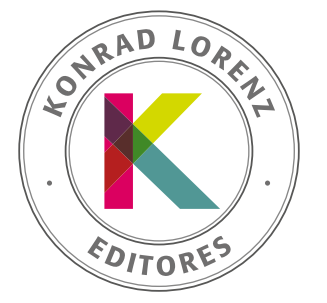

\title{
Propiedades psicométricas de la versión reducida de la Escala de Mitos sobre el Amor en una muestra de estudiantes colombianos
}

\author{
Enrique Bonilla Algovia ${ }^{a, *}$ y Esther Rivas Rivero ${ }^{a}$
}

a Universidad de Alcalá, Área de Psicología Social, Alcalá de Henares, Madrid, España

Recibido el 16 de agosto de 2018; aceptado el 6 de diciembre de 2018

PALABRAS CLAVE

amor romántico, mitos del amor romántico, género

\section{KEYWORDS}

Romantic love, love myths, gender

\begin{abstract}
Resumen El objetivo del estudio es evaluar las propiedades psicométricas de la versión reducida de la Escala de Mitos sobre el Amor en una muestra de estudiantes colombianos. Participaron 443 estudiantes con una edad media de 20.64 años. El análisis factorial confirmatorio reporta un ajuste adecuado para un modelo bifactorial. Las dos dimensiones coinciden con la literatura y tienen una fiabilidad apropiada: idealización del amor y vinculación amor-maltrato. Los resultados, al comparar las puntuaciones de mujeres y hombres, muestran diferencias estadísticamente significativas. Se concluye que el instrumento es válido y fiable para evaluar los mitos románticos en el contexto colombiano.

(C) 2018 Fundación Universitaria Konrad Lorenz. Este es un artículo Open Access bajo la licencia CC BY-NC-ND (http://creativecommons.org/licenses/bync-nd/4.0/).
\end{abstract}

Psychometric properties of the reduced version of the Myths Scale toward Love in a sample of Colombian students

Abstract The main aim of this study is to assess the psychometric properties of the reduced version of the Myths Scale toward Love in a sample of Colombian students. The research sample consists of 443 university students and their mean age is 20.64 years old. The confirmatory factor analysis shows an adequate fit for a two-factor model. Both dimensions correspond to the literature and the reliability is appropriate: Idealized love and Relation between abuse and love. The results show statistically significant differences between men and women. In conclusion, this paper verifies the validity and reliability of this scale in the Colombian background. (c) 2018 Fundación Universitaria Konrad Lorenz. This is an open access article under the CC BY-NCND license (http://creativecommons.org/licenses/bync-nd/4.0/).

* Autor para correspondencia.

Correo electrónico: enrique.bonilla@uah.es. 
El amor tiene una gran importancia en los comportamientos humanos y en la vida de las personas (Giráldez \& Sueiro, 2015); sin embargo, hasta hace poco tiempo, no ha sido considerado un tema de interés para las ciencias sociales (García \& Cedillo, 2011). La psicología ha sido, probablemente, la disciplina que lo ha abordado en mayor proporción (Esteban \& Távora, 2008). A finales del siglo XX recibió el interés de la psicología social (Sangrador, 1993), centrándose la atención en temáticas como el amor romántico y la atracción interpersonal (Yela, 1998). Desde entonces han surgido diferentes teorías sobre el tema, en las que el amor ha sido analizado como un concepto global o como un concepto multidimensional (Brenlla, Brizzio, \& Carreras, 2004). En este sentido, el estudio del discurso romántico a través de pruebas estandarizadas y validadas puede desvelar el papel que desempeña en la relaciones de pareja.

En cada etapa de la historia, al amor se le han asignado unos contenidos diferentes (Lagarde, 2001). Los cambios económicos, políticos, sociales, culturales y de pensamiento influyen en las concepciones sobre el amor (Rocha, Avendaño, Barrios, \& Polo, 2017). Es decir, se trata de un constructo social cuyo significado e implicaciones varían en función del periodo histórico, del sistema social y de la cultura. La relación entre los conceptos de amor, sexo y matrimonio ha ido variando a lo largo del tiempo (Barrón, Martínez-Íñigo, De Paúl, \& Yela, 1999). En la actualidad, el amor normativo de la cultura occidental consiste en la unión de estos tres conceptos (Yela, 2000). Esta investigación se va a centrar en lo que se conoce como "la otra cara del amor" normativo, es decir, aquellos elementos del amor que tienen consecuencias negativas: sufrimiento, frustración, problemas, idealización, mitos románticos, etc. (Yela, 2003).

El amor romántico está relacionado con una serie mitos que se transmiten a través de la socialización en los diferentes espacios (Ferrer \& Bosch, 2013; Rodríguez, Lameiras, \& Carrera, 2015; Pascual, 2016), puesto que la cultura aprueba o desaprueba los comportamientos amorosos dependiendo del lugar, del momento y del género de la persona (Rodríguez-Santero, García-Carpintero, \& Porcel, 2017). Un mito es una creencia formulada de tal modo que parece una verdad absoluta (Bosch et al., 2008; Ferrer, Bosch, \& Navarro, 2010). Los mitos del amor romántico son definidos como el conjunto de creencias que se comparten socialmente sobre la "verdadera" naturaleza del amor (Yela, 2003) y representan el imaginario social acerca del amor y su significado (Bonilla-Algovia \& Rivas-Rivero, 2018). La literatura académica clasifica los mitos románticos en mito de la media naranja, mito de la equivalencia, mito de la exclusividad, mito de la perdurabilidad, mito de la omnipotencia, mito de la fidelidad, mito del matrimonio, mito del emparejamiento, mito del libre albedrío y mito de los celos (Barrón et al., 1999; Ferrer et al., 2010; Yela, 2000, 2003).

La interiorización de los mitos del amor romántico no solo se ha relacionado con la reproducción de las desigualdades de género, sino también con la violencia contra las mujeres (Blanco, 2014; Cubells \& Calsamiglia, 2015; Ferrer \& Bosch, 2013; Marroquí \& Cervera, 2014; Pérez Grande, 2007; Power, Koch, Kralik, \& Jackson, 2006). En el proceso de socialización se transmiten los valores, conceptos y reglas patriarcales de las relaciones interpersonales y amorosas (Ferrer, Bosch, Navarro, Ramis, \& García, 2008), reproduciendo de esta manera las relaciones de pareja caracterizadas por la desigualdad, el dominio y la dependencia (Rodríguez-Castro,
Lameiras-Fernández, Carrera-Fernández, \& Vallejo-Medina, 2013). Las creencias distorsionadas sobre el amor y los mitos románticos pueden influir en la justificación y en la tolerancia a la violencia en las relaciones de pareja (Bonilla, Rivas, \& Vázquez, 2017), así como en su invisibilización (Pazos, Oliva, \& Hernando, 2014). Este escenario ha hecho que la comunidad científica centre la atención en los mitos del amor romántico y se desarrollen instrumentos estandarizados que permitan profundizar en su conocimiento; sin embargo, desde una perspectiva transcultural, apenas se ha estudiado su aceptación en diversos contextos.

A partir de la teoría de los mitos del amor romántico, surge en España la Escala de Mitos sobre el Amor (Bosch et al., 2008). En primera instancia, estaba formada por 10 ítems pero, al analizar las propiedades psicométricas, dos de ellos fueron eliminados por no adecuarse a los estándares, logrando un alfa de Cronbach de .51 en la escala de 8 ítems. Para comprobar la estructura del instrumento, las autoras realizaron un análisis factorial exploratorio y analizaron la fiabilidad de las dimensiones obtenidas. Los análisis mostraron dos factores con buenas propiedades: Idealización del amor ( $\alpha=.525)$ y Vinculación amor-maltrato $(\alpha=$ .645). Rodríguez-Castro et al. (2013), mediante modelos de ecuaciones estructurales pusieron a prueba la dimensionalidad de la escala en una muestra de adolescentes españoles. Tras comparar varios modelos obtuvieron la versión reducida de la escala de mitos hacia el amor. Esta consta de siete ítems y se agrupa en las mismas dimensiones. Estudios realizados recientemente, tanto con población general (Bosch et al., 2008; Ferrer et al., 2010) como con jóvenes y adolescentes (Bonilla-Algovia \& Rivas-Rivero, 2018; Giráldez \& Sueiro, 2015; Marroquí \& Cervera, 2014; Rodríguez-Castro et al., 2013; Rodríguez et al., 2015), demuestran la vigencia de los mitos románticos. Sin embargo, no se han encontrado estudios que analicen la validez de esta escala en el contexto colombiano. Por lo tanto, el objetivo del presente estudio es conocer las propiedades psicométricas de la versión reducida de la escala de mitos hacia el amor en una muestra de estudiantes de Colombia y analizar las diferencias en la aceptación de los mitos en función del género.

\section{Método}

\section{Participantes}

En la investigación participaron 443 estudiantes de diferentes universidades colombianas. El $71.6 \%$ eran mujeres y el $28.4 \%$ hombres. La edad media fue de 20.67 años (DT $=4.41)$ en los hombres y $20.62(D T=3.70)$ en las mujeres. Los hombres tuvieron su primera relación de pareja a los 15.66 años $(D T=2.769)$ y las mujeres a los 15.40 años (DT = 1.891) $(t=.944, p=.346)$. Una mayor cantidad de mujeres (57.7\%) que de hombres (46\%) tenía pareja en el momento de la realización de la entrevista $\left(\chi^{2}=4.973, p=.026\right)$. En la tabla 1 se muestran las principales características de los y las participantes de la investigación.

\section{Procedimiento}

La metodología de la investigación ha sido cuantitativa y transversal. En primer lugar, se informó a las universidades 
Tabla 1 Características de la muestra

\begin{tabular}{|c|c|c|c|}
\hline & $n$ & $\%$ & Media (DT) \\
\hline \multicolumn{4}{|l|}{ Género } \\
\hline Masculino & 126 & $28.4 \%$ & \\
\hline Femenino & 317 & $71.6 \%$ & \\
\hline Edad media & & & $20.64(3.911)$ \\
\hline \multicolumn{4}{|l|}{ Situación sentimental } \\
\hline Con pareja & 241 & $54.4 \%$ & \\
\hline Sin pareja & 202 & $45.6 \%$ & \\
\hline Edad a la que se tuvo pareja por primera vez & & & $15.48(2.173)$ \\
\hline \multicolumn{4}{|l|}{ Situación económica de la familia } \\
\hline Trabaja uno de los progenitores & 166 & $37.5 \%$ & \\
\hline Trabajan ambos & 246 & $55.5 \%$ & \\
\hline Trabajan otros familiares & 16 & $3.6 \%$ & \\
\hline No trabaja nadie & 15 & $3.4 \%$ & \\
\hline
\end{tabular}

de los objetivos de la investigación y se solicitó permiso. En segundo lugar, como técnica de recogida de datos, se utilizó un cuestionario autoaplicado de preguntas cerradas para su posterior tratamiento estadístico. La realización del cuestionario era voluntaria y los datos se procesaron de forma anónima.

\section{Instrumentos}

El instrumento estuvo compuesto por los siguientes apartados:

1. Características sociodemográficas y de contexto: género, edad, situación sentimental, edad a la que tuvo la primera pareja, clase social, etc.

2. Inventario de sexismo ambivalente (ASI, Glick \& Fiske, 1996), en la versión reducida y en español (Rodríguez, Lameiras, \& Carrera, 2009). Esta versión del ASI está formada por dos factores y 12 ítems. El formato de respuesta es de tipo Likert de de 0 a 5 puntos. Una mayor puntuación indica una mayor aceptación del sexismo. Los dos factores que conforman la escala son: sexismo hostil (SH) y sexismo benévolo (SB). La fiabilidad obtenida ha sido .825 .

3. Escala de Mitos hacia el Amor (Bosch et al., 2008), en la versión reducida y validada en una muestra de adolescentes españoles (Rodríguez-Castro et al., 2013). Esta versión está formada por siete ítems y dos dimensiones. La escala tiene un formato de respuesta tipo Likert de 1 (completamente en desacuerdo) a 5 (completamente de acuerdo). El factor 1, idealización del amor, está formado por cinco ítems: 1) en alguna parte hay alguien predestinado para cada persona ("tu media naranja), 2) la pasión intensa de los primeros tiempos de una relación debería durar siempre, 3) el amor es ciego, 6) los celos son una prueba de amor, y 10) el amor verdadero lo puede todo. El factor 2, vinculación amor-maltrato, está compuesto por 2 ítems: 8) se puede amar a alguien a quien se maltrata, y 9) se puede maltratar a alguien a quien se ama. En este trabajo se ha mantenido la numeración original de los ítems.

\section{Análisis}

Los datos se procesaron con el programa estadístico SPSS (IBM SPSS Statistics 22.0). En primer lugar, se estudió la normalidad de la distribución muestral y se realizó un análisis factorial exploratorio (AFE). En segundo lugar, utilizando la metodología SEM (structural equation models), se pusieron a prueba distintos modelos mediante un análisis factorial confirmatorio (AFC). El AFC se realizó con el software AMOS (IBM AMOS 24.0). Ante la falta de normalidad multivariada, analizada mediante el test de Mardia (Mardia, 1974), se empleó como técnica de estimación la de mínimos cuadrados generalizados (GLS). Para determinar el ajuste global del modelo se tuvieron en cuenta las siguientes medidas de calidad del ajuste: $\chi^{2} / g l$ menor que 3 (Escobedo, Hernández, Estebané,\& Martínez, 2016; Ruiz, Pardo, \& San Martín, 2010); CFI mayor que .90 (Cupani, 2012); RMSEA menor que .08 (Ruiz et al., 2010); GFI y AGFI igual o mayor que .90 (Escobedo et al., 2016); RMSR menor que .08. En tercer lugar, se analizaron las propiedades psicométricas del modelo utilizando las siguientes pruebas: $\bar{x}$ (media), Sx (desviación estándar), $r_{\text {item-total }}{ }^{c}$ (correlación ítem total corregida), a-ítem (alfa, si se suprime el elemento), a (alfa de Cronbach) y la fiabilidad compuesta. En cuarto y último lugar, se compararon las puntuaciones obtenidas en función del género mediante la prueba T de Student para muestras independientes. Para considerar un resultado estadísticamente significativo se adoptó la probabilidad de cometer un error tipo I de $p \leq .05$.

\section{Resultados}

La prueba KMO (Kaiser-Meyer-Olkin) y el test de esfericidad de Bartlett evaluaron la aplicabilidad del análisis 
factorial. Los estadísticos muestran una adecuación muestral aceptable $(\mathrm{KMO}=.656$; Bartlett $=572.98, p=.000)$, por lo que se procedió a realizar el AFE de la versión reducida de la escala de mitos sobre el amor. Utilizando como método de extracción mínimos cuadrados generalizados, se encontraron dos componentes con autovalores iniciales mayores a uno que acumulaban el $55.59 \%$ de la varianza. La matriz factorial rotada - utilizando como método de rotación Varimax con normalización Kaiser- convergió en 3 iteraciones. En las sumas de rotación de cargas al cuadrado, el factor 1 explicó el $21.77 \%$ de la varianza y el factor 2, el $20.68 \%$ (varianza total acumulada $=42.44 \%$ ). En la tabla 2 pueden verse la matriz de componentes rotada y las saturaciones de los ítems. Todos obtuvieron pesos factoriales superiores a .30. La distribución bifactorial coincidió con la obtenida en España (Rodríguez-Castro et al., 2013): idealización del amor (factor 1) y vinculación amor-maltrato (factor 2).

Antes de llevar a cabo el AFC se estudió la normalidad univariante y multivariante de los datos. La tabla 3 muestra los resultados de las pruebas de normalidad univariante. Los resultados de la prueba de Kolmogorov-Smirnov indican que la distribución se aleja de la normalidad. Por otra parte, la normalidad multivariada fue medida mediante el test de Mardia (Mardia, 1974) y su valor vuelve a reiterar la falta de normalidad (coeficiente Mardia $=14.422$ ). En este sentido, al realizar el análisis factorial confirmatorio se utilizó la técnica GLS como método de extracción.

El AFE reportó una estructura de dos factores, sin embargo, el ítem 3 tenía un peso factorial relativamente bajo (.342), por lo que se decidió estudiar su eliminación y poner a prueba las siguientes estructuras: modelo de un factor, modelo de dos factores con todos los ítems, modelo de dos factores sin el ítem 3. La tabla 4 expone los resultados de las pruebas de bondad de ajuste absoluto, incremental y de parsimonia del AFC. Los grados de libertad son superiores a 0 , de modo que se trata de un modelo sobreidentificado. El mejor ajuste global es el que se produce en el modelo de dos factores completo, de modo que se decidió mantener todos los ítems y no eliminar el ítem 3. La chi-cuadrado normada es inferior a 3, el error cuadrático medio de aproximación y el residuo cuadrático medio son inferiores a .80 y los índices de ajuste comparativo y de bondad de ajuste son superiores a .90 .

Tabla 2 Matriz factorial rotada

\begin{tabular}{lc}
\hline & Factor 1 \\
\hline 1. En alguna parte hay alguien predestinado para cada persona (“tu media naranja”) & .697 \\
2. La pasión intensa de los primeros tiempos de una relación debería durar siempre & .630 \\
10. El amor verdadero lo puede todo & .589 \\
6. Los celos son una prueba de amor & .416 \\
3. El amor es ciego & .342 \\
8. Se puede amar a alguien a quien se maltrata & .958 \\
9. Se puede maltratar a alguien a quien se ama & .676 \\
\hline
\end{tabular}

Tabla 3 Pruebas de normalidad univariante

\begin{tabular}{|c|c|c|c|c|c|c|}
\hline & \multicolumn{2}{|c|}{ Asimetría } & \multicolumn{2}{|c|}{ Curtosis } & \multicolumn{2}{|c|}{ Kolmogorov-Smirnov } \\
\hline & Est. & e & Est. & e & Est. & Sig. \\
\hline $\begin{array}{l}\text { 1. En alguna parte hay alguien predestinado para cada persona } \\
\text { ("tu media naranja") }\end{array}$ & -.08 & .116 & -.78 & .231 & .200 & .000 \\
\hline $\begin{array}{l}\text { 2. La pasión intensa de los primeros tiempos de una relación debería } \\
\text { durar siempre }\end{array}$ & -.60 & .116 & -.34 & .231 & .250 & .000 \\
\hline 3. El amor es ciego & -.21 & .116 & -.88 & .231 & .190 & .000 \\
\hline 6. Los celos son una prueba de amor & 1.11 & .116 & .88 & .231 & .266 & .000 \\
\hline 8. Se puede amar a alguien a quien se maltrata & 1.78 & .116 & 2.53 & .231 & .390 & .000 \\
\hline 9. Se puede maltratar a alguien a quien se ama & 1.79 & .116 & 2.32 & .231 & .394 & .000 \\
\hline 10. El amor verdadero lo puede todo & -.14 & .116 & -.95 & .231 & .176 & .000 \\
\hline
\end{tabular}

Nota: Est. = estadístico; e = error estándar; Sig. = significación.

Tabla 4 Estadísticos de bondad de ajuste de los modelos analizados

\begin{tabular}{lccccccccc}
\hline & $\chi 2$ & $g l$ & $p$ & $\chi^{2} / g l$ & GFI & CFI & AGFI & RMSEA & RMSR \\
\hline Modelo 1 factor & 115.609 & 14 & .000 & 8.258 & .925 & .564 & .851 & .128 & .169 \\
Modelo 2 factores (6 ítems, sin el 3) & 33.078 & 8 & .000 & 4.135 & .975 & .892 & .935 & .084 & .063 \\
Modelo 2 factores (7 ítems) & 34.730 & 13 & .001 & 2.672 & .978 & .907 & .952 & .061 & .056 \\
\hline
\end{tabular}


La figura 1 muestra el path diagram con los pesos de regresión estandarizados. Los estimadores son significativos en los siete ítems $(p \leq .01)$, lo que indica un buen ajuste del modelo estructural. Las cargas son superiores a .35 en todos los ítems, por lo que se confirma la validez de constructo de la estructura bifactorial de 7 ítems: idealización del amor (ítems 1, 2, 3, 6 y 10) y vinculación amor-maltrato (ítems 8 y 9).

Las propiedades psicométricas de la versión reducida de la escala de mitos hacia el amor se analizan en la tabla 5. Las medias y las desviaciones típicas son más altas en el primer factor que en el segundo. Las puntuaciones en la correlación ítem-total corregida son superiores a .30 en todos los casos, menos en el ítem 3, que tiene un valor de .29. No obstante, tal y como se verificó anteriormente, el ajuste al modelo es mejor si se mantiene el ítem. Además, la fiabilidad total de la escala $(\alpha=.64)$ apenas mejoraría con su eliminación. El alfa de Cronbach de la subescala de idealización del amor es .662 (hombres, $\alpha=$.714; mujeres, $\alpha=.643$ ) y el de la subescala de vinculación amor-maltrato es .786 (hombres, $\alpha=.793$; mujeres, $\alpha=.765$ ). Para evaluar el ajuste del modelo de medida se ha analizado la fiabilidad compuesta de cada uno de los constructos. Esta ha sido .69 en la subescala idealización del amor y .77 en la subescala vinculación amor-maltrato.

En la tabla 6 se analizan las correlaciones de Pearson entre los 7 ítems que componen la escala. Las correlaciones son significativas entre los ítems del mismo factor, es decir, los ítems del primer factor se correlacionan significativamente entre sí y los ítems del segundo factor también. No ocurre lo mismo entre los ítems de diferente factor, en los que la correlación es no significativa (salvo en el ítem 6). En general, los valores son bajos y positivos. La única correlación moderada (.649) es la que se produce entre los ítems 8 y 9 , que pertenecen a la misma subescala (vinculación amor-maltrato).

La validez externa se ha evaluado mediante las correlaciones de Pearson entre la escala de mitos hacia el amor y el inventario de sexismo ambivalente (véase tabla 7). Tanto el factor idealización del amor como el factor vinculación amor-maltrato correlacionan significativamente con el sexismo ambivalente, el sexismo hostil y el sexismo benévolo.

Al comparar las puntuaciones de mujeres y hombres, los resultados ponen de manifiesto diferencias estadísticamente significativas en el acuerdo con varios mitos románticos (véase tabla 8). Los hombres obtienen mayores puntuaciones medias que las mujeres en el ítem $6(t=3.946$, $p=.000)$, en el ítem $8(t=3.619, p=.000)$ y en el ítem 9 $(t=3.428, p=.001)$. En cuanto a los resultados totales de cada una de las subescalas, los datos reportan una mayor aceptación de la idealización del amor $(M=2.93, D T=.742)$ que de la vinculación amor-maltrato $(M=1.55, D T=.852)$. La media de la subescala idealización del amor es muy similar en hombres $(M=2.96, D T=.801)$ y en mujeres $(M=2.91$, $D T=.718)(t=.671, p=.503)$. En cambio, la subescala vinculación amor-maltrato reporta diferencias estadísticamente significativas, siendo los hombres quienes más puntúan $\left(M_{\text {hombres }}=1.83, D T=1.011 ; M_{\text {mujeres }}=1.44, D T=.754 ; t=\right.$ $3.873, p=.000)$.

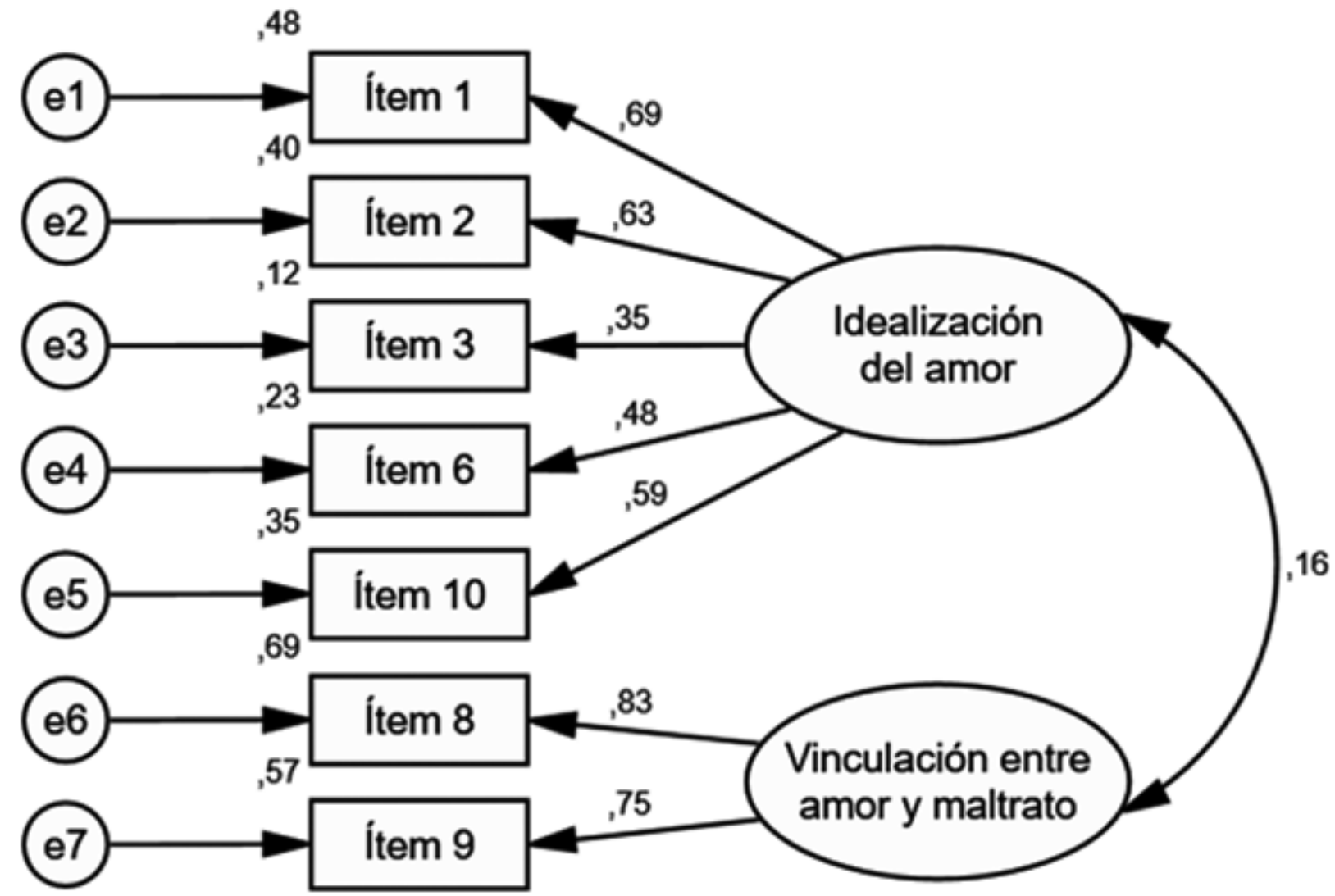

Figura 1. Diagrama estructural del análisis factorial confirmatorio 
Tabla 5 Propiedades psicométricas de los ítems por subescalas

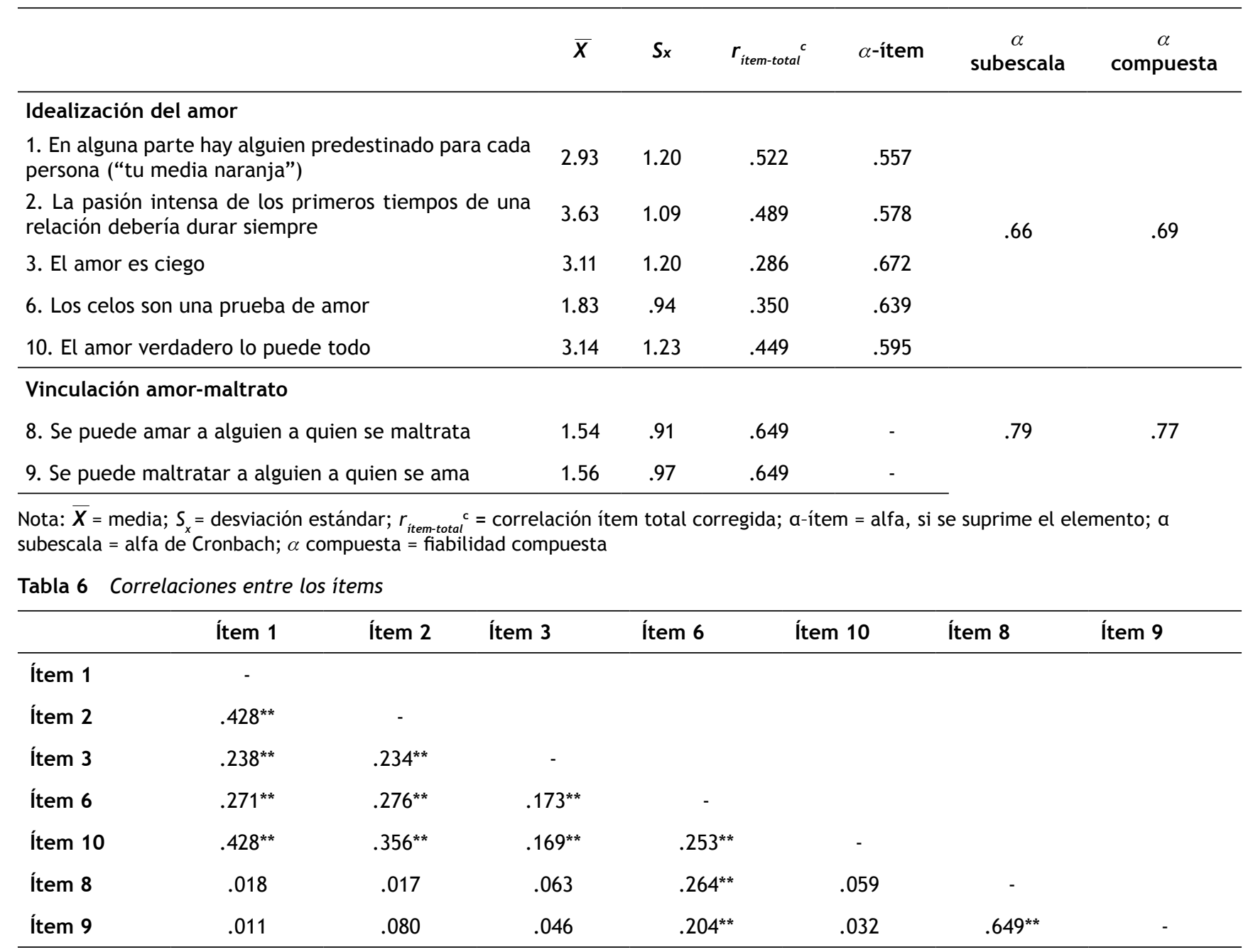

Nota: ${ }^{*} p \leq .05 ;{ }^{* *} p \leq .01$

Tabla 7 Matriz de correlaciones entre los mitos del amor romántico y el sexismo ambivalente

\begin{tabular}{|c|c|c|c|}
\hline & ASI & SH & SB \\
\hline Subescala de idealización del amor & $.528^{* *}$ & $.288^{* *}$ & $.585^{* *}$ \\
\hline Subescala de vinculación amor-maltrato & $.239 * *$ & $.252^{* *}$ & $.135^{* *}$ \\
\hline
\end{tabular}

Nota: ${ }^{*} p \leq .05 ;{ }^{* *} p \leq .01 ; \mathrm{ASI}=$ sexismo ambivalente; $\mathrm{SH}=$ sexismo hostil; $\mathrm{SB}=$ sexismo benévolo.

\section{Discusión}

Las concepciones sobre el amor tienen una gran repercusión en la vida de las personas, puesto que validan o invalidan las cogniciones, los comportamientos y los sentimientos relativos a las relaciones de pareja. En el ámbito iberoamericano, partiendo de diferentes teorías y escalas (Hendrick \& Hendrick, 1986; Lee, 1977; Sternberg, 1986), se han realizado recientemente numerosos estudios sobre los componentes, los estilos y las actitudes hacia el amor: en España (Ferrer et al., 2008; Rodríguez et al., 2015; Rodríguez-Santero et al., 2017), México (Mazadiego \& Garcés, 2011; Xóchitl, Sánchez, \& Robles, 2013), Perú (Lascurain, Lavandera,\& Manzanares, 2017), Bolivia (Cooper \& Pinto,
2008), Argentina (Brenlla et al., 2004; Camacho et al., 2012), Chile (Maureira \& Maureira, 2012), Colombia (Caycedo et al., 2007; Rocha et al., 2017). Sin embargo, a pesar de la existencia de investigaciones sobre los estilos y componentes amorosos, faltan estudios que se centren específicamente en los mitos del amor romántico, sobre todo en el contexto latinoamericano, por lo que se hace necesario su análisis científico. Esto es especialmente relevante teniendo en cuenta la prevalencia de la violencia de género en la región. Ante esto, y dada la importancia de obtener información a través de instrumentos validados (Gómez-Lugo, Saavedra-Roa, Pérez-Durán, \& Vallejo-Medina, 2016), el presente trabajo se ha centrado en analizar las propiedades psicométricas de la versión reducida de la escala de mitos 
Tabla 8 Diferencias estadísticamente significativas según género

\begin{tabular}{|c|c|c|c|c|c|}
\hline & \multicolumn{2}{|c|}{ Hombres } & \multicolumn{2}{|c|}{ Mujeres } & \multirow{2}{*}{ TdeStuden } \\
\hline & M & DT & M & DT & \\
\hline \multicolumn{6}{|l|}{ Mito idealización del amor } \\
\hline $\begin{array}{l}\text { 1. En alguna parte hay alguien predestinado para cada persona ("tu } \\
\text { media naranja") }\end{array}$ & 2.79 & 1.25 & 2.98 & 1.17 & -1.551 \\
\hline $\begin{array}{l}\text { 2. La pasión intensa de los primeros tiempos de una relación debería } \\
\text { durar siempre }\end{array}$ & 3.68 & 1.14 & 3.61 & 1.07 & 668 \\
\hline 3. El amor es ciego & 3.17 & 1.13 & 3.09 & 1.23 & 681 \\
\hline 6. Los celos son una prueba de amor & 2.13 & 1.07 & 1.71 & .86 & $3.946^{* * *}$ \\
\hline 10. El amor verdadero lo puede todo & 3.05 & 1.27 & 3.17 & 1.21 & -.972 \\
\hline \multicolumn{6}{|l|}{ Mito vinculación amor-maltrato } \\
\hline 8. Se puede amar a alguien a quien se maltrata & 1.81 & 1.06 & 1.43 & .81 & $3.619^{* * *}$ \\
\hline 9. Se puede maltratar a alguien a quien se ama & 1.84 & 1.16 & 1.45 & .87 & $3.428^{* * *}$ \\
\hline
\end{tabular}

Nota: ${ }^{*} p \leq .05 ;{ }^{* *} p \leq .01 ;{ }^{* * *} p \leq .001$

hacia el amor en una muestra de estudiantes universitarios de Colombia.

La dimensionalidad de la escala ha quedado confirmada mediante la metodología de ecuaciones estructurales (SEM). Las pruebas de bondad de ajuste absoluto, incremental y de parsimonia reportaron que el modelo con mejor ajuste era el bifactorial de 7 ítems $\left(\chi^{2} / g l=2.67\right.$; GFI = .98; $\mathrm{CFI}=.91 ; \mathrm{AGFI}=.95 ; \mathrm{RMSEA}=.06$; RMSR= .06). Todos los estadísticos cumplían con los criterios establecidos previamente (Cupani, 2012; Escobedo et al., 2016; Ruiz et al., 2010) y eran mejores que los de los modelos alternativos. Los estimadores fueron significativos en los 7 ítems y los pesos de regresión estandarizada del path diagram oscilaron entre .35 y .83. El modelo definitivo está formado por dos dimensiones que coinciden con la estructura obtenida en la validación española (Rodríguez-Castro et al., 2013): idealización del amor (5 ítems) y vinculación amor-maltrato ( 2 ítems). Los coeficientes del alfa de Cronbach y la fiabilidad compuesta obtenidos fueron superiores a .60 en ambas subescalas, por lo que la fiabilidad del instrumento se encuentra dentro del rango de aceptabilidad (Hair, Anderson, Tatham, \& Black, 1999). Además, estos coeficientes son más altos que los hallados en otros estudios realizados en el ámbito internacional (Bonilla-Algovia \& Rivas-Rivero, 2018; Bosch et al., 2008). Las pruebas de validez externa han confirmado que existe una asociación significativa entre los mitos del amor romántico y el sexismo ambivalente (Glick y Fiske, 1996). Estudios realizados en España reportan esta asociación, esto es, a mayor aceptación de los mitos románticos, mayores niveles de sexismo hostil y sexismo benévolo (Rodríguez et al., 2015; Rodríguez-Castro et al., 2013). En síntesis, a la vista de las pruebas llevadas a cabo, la versión reducida de la escala de mitos hacia el amor es una herramienta válida y fiable para medir los mitos del amor romántico en el contexto colombiano.

La interiorización de los mitos del amor romántico varía en función del componente. La idealización del amor está más aceptada que la vinculación amor-maltrato. En cuanto a los resultados en función del género, no se observan diferencias estadísticamente significativas en la idealización del amor, pero sí en la vinculación amor-maltrato, donde los hombres obtienen puntuaciones medias más altas que las mujeres. Estos resultados han sido obtenidos en otros países con muestras de diferentes edades (Bonilla-Algovia \& Rivas-Rivero, 2018; Bosch et al., 2008; Rodríguez et al., 2015; Rodríguez-Castro et al., 2013). El análisis individual de los ítems reitera las diferencias en función del género en la visión del amor. Los hombres están más de acuerdo que las mujeres con los mitos relacionados con el maltrato ("se puede amar a alguien a quien se maltrata" y "se puede maltratar a alguien a quien se ama") y con el mito de los celos ("los celos son una prueba de amor"), lo que podría derivar en altos niveles de violencia contra la pareja (Martínez-León, Mathes, Avendaño, Peña, \& Sierra, 2018). Las mujeres, aunque a este respecto no se hallaron diferencias estadísticamente significativas, están más de acuerdo con el mito de la media naranja ("en alguna parte hay alguien predestinado para cada persona") y con el mito de la omnipotencia ("el amor verdadero lo puede todo"). Por lo tanto, los resultados muestran que mujeres y hombres tienen diferentes concepciones acerca del amor y los mitos románticos, siendo estos quienes presentan una visión más distorsionada y vinculada al maltrato en comparación con las mujeres. Las diferencias entre mujeres y hombres a la hora de entender el amor estarían relacionadas con la socialización diferencial o socialización de género (Ferrer \& Bosch, 2013; Pascual, 2016). Hombres y mujeres son socializados en estilos amorosos distintos, vinculados a los estereotipos de género, que perpetúan las relaciones de pareja basadas en modelos patriarcales (Bonilla-Algovia \& Rivas-Rivero, 2018). Por lo tanto, la interiorización de los mitos románticos contribuye a reproducir las desigualdades en las relaciones de pareja y aumenta la tolerancia a la violencia (Blanco, 2014; Bonilla et al., 2017; Ferrer et al., 2010), de manera que es necesario sensibilizar a la sociedad y fomentar estilos amorosos alejados del maltrato.

La investigación presenta ciertas limitaciones. Primero, en la escala de mitos hacia el amor, el modelo bifactorial de siete ítems es el que muestra mejores indicadores de validez y bondad de ajuste, lo que no quiere decir que no existan 
otros modelos con propiedades psicométricas adecuadas. Segundo, el tamaño de la muestra supera el recomendado para realizar la validación de un instrumento, pero no es una muestra representativa de toda la población colombiana. Y, por último, el estudio está basado en una metodología de corte transversal que no permite analizar la evolución y las implicaciones de las creencias sobre el amor a lo largo del tiempo.

En conclusión, las creencias sobre el amor y su relación con otros elementos como la violencia son ámbitos de estudio emergentes, y en Colombia se carece de datos al respecto. Este trabajo pone de manifiesto la validez de un instrumento que permite profundizar en el análisis de los mitos del amor romántico y poner en marcha acciones educativas y de concienciación. La prevención de la violencia de género y la coeducación son las mejores herramientas para fomentar modelos alternativos de amor basados en el respeto, la igualdad y la no violencia. La escala de mitos hacia el amor es una herramienta útil para analizar las concepciones sobre el amor romántico y obtener información que permita implementar programas de intervención en el contexto colombiano.

\section{Agradecimientos}

Este trabajo se realizó con el apoyo de un contrato predoctoral de formación de profesorado universitario de la Universidad de Alcalá.

\section{Referencias}

Barrón, A., Martínez-Íñigo, D., De Paúl, P., \& Yela, C. (1999). Romantic beliefs and myths in Spain. The Spanish Journal of Psychology, 2, 64-73. http://dx.doi.org/10.1017/S1138741600005461

Blanco, M. A. (2014). Implicaciones del uso de las redes sociales en el aumento de la violencia de género en adolescentes. Comunicación y Medios, 30, 124-141.

Bonilla, E., Rivas, E., \& Vázquez, J. J. (2017). Tolerancia y justificación de la violencia en relaciones de pareja adolescentes. Apuntes de Psicología, 35, 55-61.

Bonilla-Algovia, E., \& Rivas-Rivero, E. (2018). Mitos del amor romántico en una muestra de futuros y futuras docentes. Revista de Estudios e Investigación en Psicología y Educación, 5, 113117. http://dx.doi.org/10.17979/reipe.2018.5.2.3624

Bosch, E., Ferrer, V. A., García, M. E., Ramis, M. C., Mas, M. C., Navarro, C., \& Torrens, G. (2008). Del mito del amor romántico a la violencia contra las mujeres en la pareja. Madrid: Instituto de la Mujer.

Brenlla, M. E., Brizzio, A., \& Carreras, A. (2004). Actitudes hacia el amor y apego. Psicodebate, 4, 7-23. http://dx.doi.org/10.18682/ pd.v4i0.491

Camacho, J. M., Regalado, P., Carrea, G., Grosso, C., Geleazzi, F., Gunther, G., Del Socorro, M., Delfino, A., \& Ramos, J. (2012). Actitudes hacia el amor y estilos de humor en mujeres y varones: ¿Nos diferencia el sexo o el género? Revista Latinoamericana de Ciencia Psicológica, 4, 13-27.

Caycedo, C., Cubides, I., Martín, A., Cortés, O. F., Berman, S., Oviedo, A. M.,\& Suárez, I. (2007). Relación entre el género y las experiencias de cortejo y actitudes hacia las relaciones románticas en adolescentes bogotanos. Psicología desde el Caribe, (20), 76-92.

Cooper, V., \& Pinto, B. (2008). Actitudes ante el amor y la teoría de Sternberg: un estudio correlacional en jóvenes universitarios de 18 a 24 años de edad. AJAYU, 6, 181-206.
Cubells, J., \& Calsamiglia, A. (2015). El repertorio del amor romántico y las condiciones de posibilidad para la violencia machista. Universitas Psychologica, 14, 1681-1694. http://dx.doi.org/10.11144/ Javeriana.upsy14-5.rarc

Cupani, M. (2012). Análisis de ecuaciones estructurales: conceptos, etapas de desarrollo y un ejemplo de aplicación. Revista Tesis (1), 186-199.

Escobedo, M. T., Hernández, J. A., Estebané, V., \& Martínez, G. (2016). Modelos de ecuaciones estructurales: características, fases, construcción, aplicación y resultados. Ciencia y Trabajo (55), 16-22. http://dx.doi.org/10.4067/S0718-24492016000100004

Esteban, M. L., \& Távora, A. (2008). El amor romántico y la subordinación social de las mujeres: revisiones y propuestas. Anuario de Psicología, 39, 59-73.

Ferrer, V. A., \& Bosch, E. (2013). Del amor romántico a la violencia de género: Para una coeducación emocional en la agenda educativa. Revista de Currículum y Formación del Profesorado, 17, 105-122.

Ferrer, V. A., Bosch, E., Navarro, C., Ramis, M. C., \& García E. (2008). El concepto de amor en España. Psicothema, 20, 589-595.

Ferrer, V. A., Bosch, E., \& Navarro, C. (2010). Los mitos románticos en España. Boletín de Psicología (99), 7-31.

García, A., \& Cedillo, P. (2011). Tras los pasos del amor: un recuento desde las ciencias sociales. Estudios Sociológicos, 29, 551-602.

Giráldez, N., \& Sueiro, E. (2015). Mitos do amor romántico. Revista de Estudios e Investigación en Psicología y Educación, Extr. (5), 57-61. http://dx.doi.org/10.17979/reipe.2015.0.05.190

Glick, P., \& Fiske, S. T. (1996). The ambivalent sexism inventory: Differentiating hostile and benevolent sexism. Journal of Personality and Social Psychology, 70(3), 491-512. http://dx.doi. org/10.1037/0022-3514.70.3.491

Gómez-Lugo, M., Saavedra-Roa, A., Pérez-Durán, C., \& Vallejo-Medina, P. (2016). Validity and reliability of a set of sexual stimuli in a sample of Colombian heterosexual young women. Suma Psicológica, 23, 109-115. http://dx.doi.org/10.1016/j.sumpsi.2016.09.001

Hendrick, C., \& Hendrick, S. (1986). A theory and method of love. Journal of Personality and Social Psychology, 50, 392-402. http://dx.doi.org/10.1037/0022-3514.50.2.392

Hair, J. F., Anderson, R. E., Tatham, R. L., \& Black, W. C. (1999). Análisis multivariante. Madrid: Prentice Hall Iberia.

Lagarde, M. (2001). Claves feministas para la negociación del amor. Managua: Puntos de Encuentro.

Lascurain, P. C., Lavandera, M. C., \& Manzanares, E. (2017). Propiedades psicométricas de la escala de actitudes sobre el amor (LAS) en universitarios peruanos. Acta Colombiana de Psicología, 20, 270281. http:/dx.doi.org/10.14718/ACP.2017.20.2.13

Lee, J. A. (1977). A typology of styles of loving. Personality and Social Psychology Bulletin, 3(2), 173-182. http://dx.doi. org/10.1177/014616727700300204

Mardia, K. V. (1974). Applications of some measures of multivariate skewness and kurtosis in testing normality and robustness studies. Sankhyā: The Indian Journal of Statistics, 36, 115-128.

Maureira, F., \& Maureira, Y. (2012). Características de los componentes del amor de pareja en una muestra de estudiantes chilenos. Revista Electrónica de Psicología Iztacala, 15, 206-217.

Marroquí, M., \& Cervera, P. (2014). Interiorización de los falsos mitos del amor romántico en jóvenes. Reidocrea, 3, 142-146.

Martínez-León, N. C., Mathes, E., Avendaño, B. L., Peña, J.J., \& Sierra, J.C. (2018). Psychometric Study of the Interpersonal Jealousy Scale in Colombian Samples. Revista Latinoamericana de Psicología, 50, 21-30. http://dx.doi.org/10.14349/rlp.2018. v50.n1.3

Mazadiego, T.J., \& Garcés, J.R.N. (2011). El amor medido por la escala triangular de Sternberg. Psicolatina, 22, 1-10.

Pascual, A. (2016). Sobre el mito del amor romántico: amores cinematográficos y educación. Dedica, Revista de Educação e Humanidades, 10 , 63-78. 
Pazos, M., Oliva, A., \& Hernando, Á. (2014). Violencia en relaciones de pareja de jóvenes y adolescentes. Revista Latinoamericana de Psicología, 46, 148-159. http://dx.doi.org/10.1016/ S0120-0534(14)70018-4

Pérez Grande, M. D. (2007). La violencia de género: prevención educativa. Papeles Salmantinos de Educación (8), 73-94.

Power, C., Koch, T., Kralik, D., \& Jackson, D. (2006). Lovestruck: Women, romantic love and intimate partner violence. Contemporary Nurse, 21, 174-185. http://dx.doi.org/10.5555/conu.2006.21.2.174

Rocha, B. L., Avendaño, C. E., Barrios, M. A., \& Polo, A. (2017). Actitudes hacia el amor en relaciones románticas de jóvenes universitarios. Praxis \& Saber, 8, 155-178. http://dx.doi.org/10.19053/22160159. v7.n15.2016.5727

Rodríguez-Castro, Y., Lameiras-Fernández, M., Carrera-Fernández, M.V., \& Vallejo-Medina, P. (2013). La fiabilidad y validez de la escala de mitos hacia el amor: las creencias de los y las adolescentes. Revista de Psicología Social, 28, 157-168. http://dx.doi.org/10.1174/021347413806196708

Rodríguez, Y., Lameiras, M., \& Carrera, M. V. (2009). Validación de la versión reducida de las escalas ASI y AMI en una muestra de estudiantes españoles. Psicogente, 12, 284-295.

Rodríguez, Y., Lameiras, M., \& Carrera, M. V. (2015). Amor y sexismo: una peligrosa relación en los y las adolescentes gallegos/as. Revista de Estudios e Investigación en Psicología y Educación, Extr. (2), 11-14. http://dx.doi.org/10.17979/reipe.2015.0.02.234
Rodríguez-Santero, J., García-Carpintero, M. A., \& Porcel, A. M. (2017). Los estilos de amor en estudiantes universitarios: diferencias en función del sexo-género. Revista Internacional de Sociología, 75, 1-13. http://dx.doi.org/10.3989/ris.2017.75.3.15.171

Ruiz, M. A., Pardo, A., \& San Martín, R. (2010). Modelos de ecuaciones estructurales. Papeles del Psicólogo, 31, 34-45.

Sangrador, J. L. (1993) Consideraciones psicosociales sobre el amor romántico. Psicothema, 5, 181-196.

Sternberg, R. J. (1986). A triangular theory of love. Psychological Review, 93(2), 119-135. http://dx.doi.org/10.1037/0033-295X.93.2.119

Xóchitl, I., Sánchez, A., \& Robles, F. J. (2013). Relaciones entre estilos de amor y violencia en adolescentes. Psicología desde el Caribe, 30, 211-235.

Yela, C. (1998). Diferencias entre sexos en los juicios verbales sobre su comportamiento amoroso y sexual. Revista de Psicología General y Aplicada, 51, 115-147.

Yela, C. (2000). El amor desde la Psicología Social: ni tan libres ni tan racionales. Madrid: Pirámide.

Yela, C. (2003). La otra cara del amor: mitos, paradojas y problemas. Encuentros en Psicología Social, 1, 263-267. 